American J. of Engineering and Applied Sciences 1 (4): 260-265, 2008

ISSN 1941-7020

(C) 2008 Science Publications

\title{
Vector Control of a Variable Reluctance Machine Stator and Rotor Discs Imbricates
}

\author{
Abdelhamid Boucetta \\ Faculty of Sciences Engineering, University of Batna, \\ Rue Chahid Mohamed El-hadi Boukhlouf, 05000 Batna, Algeria
}

\begin{abstract}
This study was devoted, in its first part, to the configuration and the modelling of the Imbricate Discs Variable Reluctance Machine (IDVRM), which was simplified with the assumption of stator structure alike to that of the synchronous machine, leading to the possibility of using the PARK's transformation. Then, a vector control strategy, which maintains a constant saturation level in the machine, was set-up. This strategy, which consists in keeping the stator direct current at a constant value, allowed the linearization of the machine model and the control was chose in the same way as a DC machine. A simulation by Matlab/Simulink of the IDVRM, fed by PWM inverter, showed the simplicity and the effectiveness of the proposed control strategy for this type of machine.
\end{abstract}

Key words: Stator and rotor discs imbricates, variable reluctance machine, pulsed width modulation (PWM) inverter, vector control

\section{INTRODUCTION}

In this study, the machine dealt with is a variable reluctance machine with imbricates discs.

This kind of machine is commonly called Machines with Synchronous Reluctance.

In fact, it is a synchronous machine with projecting poles which has trapezoidal magnetic core instead of field windings. The machine works exclusively on the torque of the variable reluctance.

Its rotor made of Epoxy insulator used for applications of very high speeds ${ }^{[1]}$.

The demand for engines with very high masstorque ratio leads to machines with multi variable reluctance with poly air-gap. This approach deals with a field which is not covered by the conventional machines. In the conventional machines (with continuous or synchronous currents), the improvement of the mass performances gets trough the supply of ampere-turns without leading to joule losses. For these reasons we can use supra-conductor materials. The advantage of the variable reluctance machine, where a high mass-torque is required (in particular at low speeds), without lowering the efficiency of the machine, are generally fed by the industrial network which imposes a sinusoidal voltage, or by inverters which deliver a square wave voltage, or current, or even by a chopper with or without modulation of impulse ${ }^{[2]}$.

This kind of machine is a subject of various studies, concerning their designs ${ }^{[1-4]}$, the optimization of their controllers, particularly in variable speeds ${ }^{[5-7] .}$
The performances and the reliability of any classical control technique depend primarily on the accuracy of the machine model, on the control strategy simplicity of the synchronous reluctance machines, on the geometrical shape of the rotor and on the weak airgap. In order to simplify the VRM modelling, the electrical equations are generally expressed in Park coordinates. However, because of the saturation and more especially of the decoupling which appears between direct and quadratic flux (saturation's effects), the Park's model becomes complicated and relatively difficult to use. The choice of the vector control, for the VRM with imbricates discs by a judicious choice of the direct current value id, can make this effect negligible and obtain a constant level of saturation in the machine for any operating point ${ }^{[6]}$.

We then get a simple model in which the electromagnetic torque is controlled by the only quadratic current iq. Then the Imbricate Discs Variable Reluctance Machine (IDVRM) control becomes similar to that of a DC machine.

In order to maintain the direct current at a reference value and be able to vary the quadratic current without any incidence, we use a decoupling algorithm ${ }^{[6]}$ which allows a separate control of the currents (direct and quadratic). By using Matlab/Simulink, the simulation of the IDVRM, fed by Pulsed Width Modulation (PWM) inverter, the simplicity and the effectiveness of the proposed control strategy is proved and discussed. 


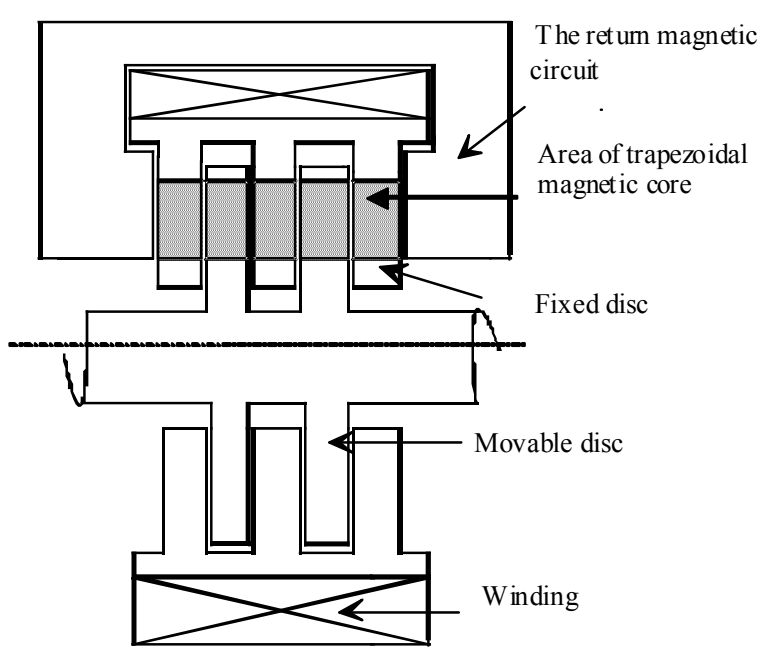

Fig. 1: Sectional view of the machine

IDVRM description: Among the many possible structures of a variable reluctance machine (VRM), poly-air-gap, a typical one is shown in (Fig. 1). These machines are made of a stacked lamination of nonmagnetic insulating Epoxy discs. Sectors made of which trapezoidal magnetic cores, a simple manufactured winding (cylindrical winding) covers all the discs. A cylinder head ensures the return of the flux .The magnetic circuit permeability varies according to the angle $\theta$.

Such a machine is primarily single-phased, however, by the assembly of several suitably shifted machines, we get a poly-phased machine.

The variable power supply of the reluctance machines is usually done by sinusoidal shape or by other shapes of voltage and current. Other forms of feeding waves can improve the machine's performances. So, this typical structure of machine is belonging to special electrical machines.

IDVRM general principals: In the discoid machines the variation of inductance is obtained by displacement of the number of discs rotor $\mathrm{Ne}$ with respect to $\mathrm{Ne}+1$ discs stator. These discs, containing a trapezoidal magnetic core, are in an axial magnetic field created by cylindrical winding. At the time of the movement of the rotor, we distinguish two significant positions (Fig. 2).

- Unaligned position: When the trapezoidal magnetic cores are symmetrically shifted inductance is minimal and the position is unstable

- Aligned position: When the trapezoidal magnetic cores of the rotor and the stator are aligned, leading to the maximisation of the inductance in which the machine position is stable

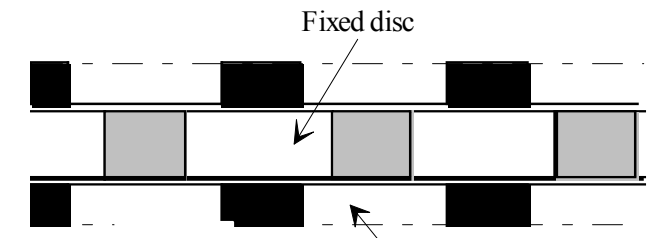

(a)

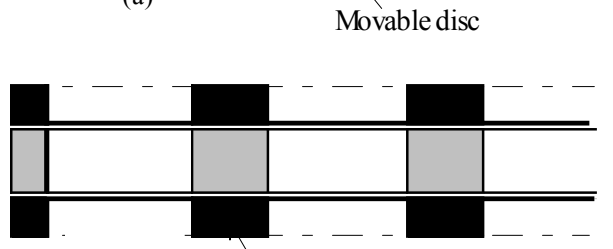

(b)

Trapezoidal magnetic core

Fig. 2: (a): Unaligned position, (b): Aligned position

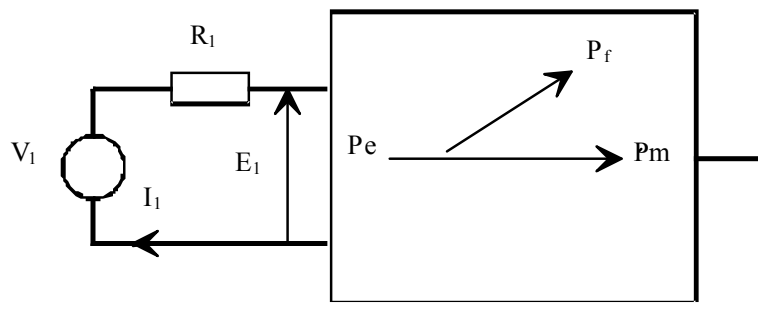

Fig. 3: Electrical model for variable reluctance machine

The determination of the variation reluctance $R$ with the position of the rotor $\theta$, by using initially sinusoidal variation reluctance (unsaturated) which has the form:

$$
\mathrm{R}(\theta)=\mathrm{R}_{0}+\mathrm{r} \cos (\kappa \theta)
$$

Where:

$\kappa=$ The number of trapezoidal magnetic core per disc

$\mathrm{r}=$ First harmonic.

$\mathrm{R}_{0}=$ Average reluctance

This rotating machine can be presented as a limped black box converting the electrical power into mechanical power. The electric power is produced by electrical source giving a mechanical power (Fig. 3).

When the losses are neglected, the energy balance is written as:

$$
d W+E_{1} I_{1} d t+F d x=0
$$

Where:

$\mathrm{W}=$ The stored magnetic energy

$\mathrm{F}=$ The force

$\mathrm{X}=$ The Position 


$$
V=R_{S} I_{S}+N_{s} \frac{d \phi}{d t}+N_{s} \frac{d \varphi}{d t}
$$

Where:

$\frac{\mathrm{d} \phi}{\mathrm{dt}}=$ The mean generated flux

$\frac{\mathrm{d} \varphi}{\mathrm{dt}}=$ The fringing flux

$\mathrm{N}_{\mathrm{S}}=$ Whorl number

$\mathrm{R}_{\mathrm{S}}=$ The stator winding resistance

From these formulae, it is clear that the general concept of this machine is related to electromagnetic laws. Therefore, it can be modelled by using the same method for modelling other alternating current machines, especially synchronous machines.

IDVRM modelling in Park's reference: In order to simplify the modelling of the machine, the following assumptions are made:

- The distribution of the magneto-motive forces is sinusoidal

- The magnetic circuit of the machine is unsaturated

- The irregularities of the air-gap to the stator notches are ignored

- The phenomena of hysteresis and the Eddy currents are neglected

The Park's reference is defined by two axes, direct and quadratic axes, which depend on the rotor position. The direct axis coincides with the axis of symmetry of the rotor teeth. This is represented by the mechanical angle $\theta$ referenced to the stator axis.

The electrical equations of the electric machines in fixed a reference tilted to the stator are described by:

$$
\left[\begin{array}{c}
v a \\
v b \\
v c
\end{array}\right]=R s \cdot\left[\begin{array}{l}
i a \\
i b \\
i c
\end{array}\right]+\frac{d}{d t}\left[\begin{array}{l}
\phi a \\
\phi b \\
\phi c
\end{array}\right]
$$

Where:

Rs $=$ The resistance of the stator phases

$\mathrm{va}, \mathrm{vb}, \mathrm{vc}=$ Stator phase voltages

$\mathrm{ia}, \mathrm{ib}, \mathrm{ic}=$ The stator phase currents

$\phi \mathrm{a}, \phi \mathrm{b}, \phi \mathrm{c}=$ The stator winding total flux $\mathrm{T}_{32}$.

Applying the restricted Concordia transformation

$$
\left[\begin{array}{c}
v \alpha \\
v \beta
\end{array}\right]=\operatorname{Rs}\left[\begin{array}{l}
i \alpha \\
i \beta
\end{array}\right]+\frac{d}{d t}\left[\begin{array}{l}
\phi \alpha \\
\phi \beta
\end{array}\right]
$$

$$
\left[\begin{array}{l}
\mathrm{x} \alpha \\
\mathrm{x} \beta
\end{array}\right]=\mathrm{T}_{32}^{\mathrm{t}}\left[\begin{array}{l}
\mathrm{xa} \\
\mathrm{xb} \\
\mathrm{xc}
\end{array}\right]
$$

$\mathrm{x}$ : Replaced by $\mathrm{v}, \mathrm{i}$ and $\phi$

$\theta$ : The electrical angle indicating the position of the rotor with respect to the stator

$\left[\begin{array}{c}\mathrm{vd} \\ \mathrm{vq}\end{array}\right]=\operatorname{Rs}\left[\begin{array}{c}\mathrm{id} \\ \mathrm{iq}\end{array}\right]+\frac{\mathrm{d}}{\mathrm{dt}}\left[\begin{array}{c}\phi \mathrm{d} \\ \phi \mathrm{q}\end{array}\right]+\left[\begin{array}{rr}0 & -1 \\ 1 & 0\end{array}\right]\left[\begin{array}{l}\phi \mathrm{d} \\ \phi \mathrm{q}\end{array}\right] \mathrm{p} \frac{\mathrm{d} \theta}{\mathrm{dt}}$

In the variable reluctance machines with sinusoidal windings distribution, like in any synchronous machine, $\phi d$ and $\phi q$ are linear functions of the currents id and iq.

$$
\begin{gathered}
\phi d=\mathrm{Ld} \mathrm{id}+\phi_{\mathrm{TMC}} \\
\phi \mathrm{q}=\mathrm{Lq} \text { iq }
\end{gathered}
$$

$\phi_{\mathrm{TMC}}=$ The flux generated by trapezoidal magnetic core

$\mathrm{Ld}, \mathrm{Lq}=$ Direct and quadratic inductances. They are supposed to be independent of $\theta$

Where:

$$
\frac{\mathrm{d} \theta}{\mathrm{dt}}=\omega=\mathrm{p} \Omega
$$

$\mathrm{p}$ is the number of pairs of poles.

The electromagnetic torque developed by variable reluctance machines with sinusoidal e.m.f. ${ }^{[5]}$ can be given by the following relation:

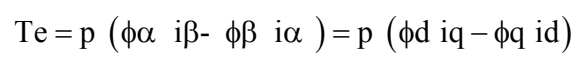

And the mechanical equation is given by:

$$
\mathrm{J} \frac{\mathrm{d} \Omega}{\mathrm{dt}}=\mathrm{Te}-\mathrm{Tch}-\kappa_{\mathrm{f}} \Omega
$$

Where:

$\mathrm{J}=$ The combined machine and load moment of inertia

$\mathrm{k}_{\mathrm{f}}=$ The combined machine and load friction coefficient

$\mathrm{Te}=$ The electromagnetic torque

$\mathrm{Tch}=$ The load torque

$\Omega=$ The rotor angular speed 


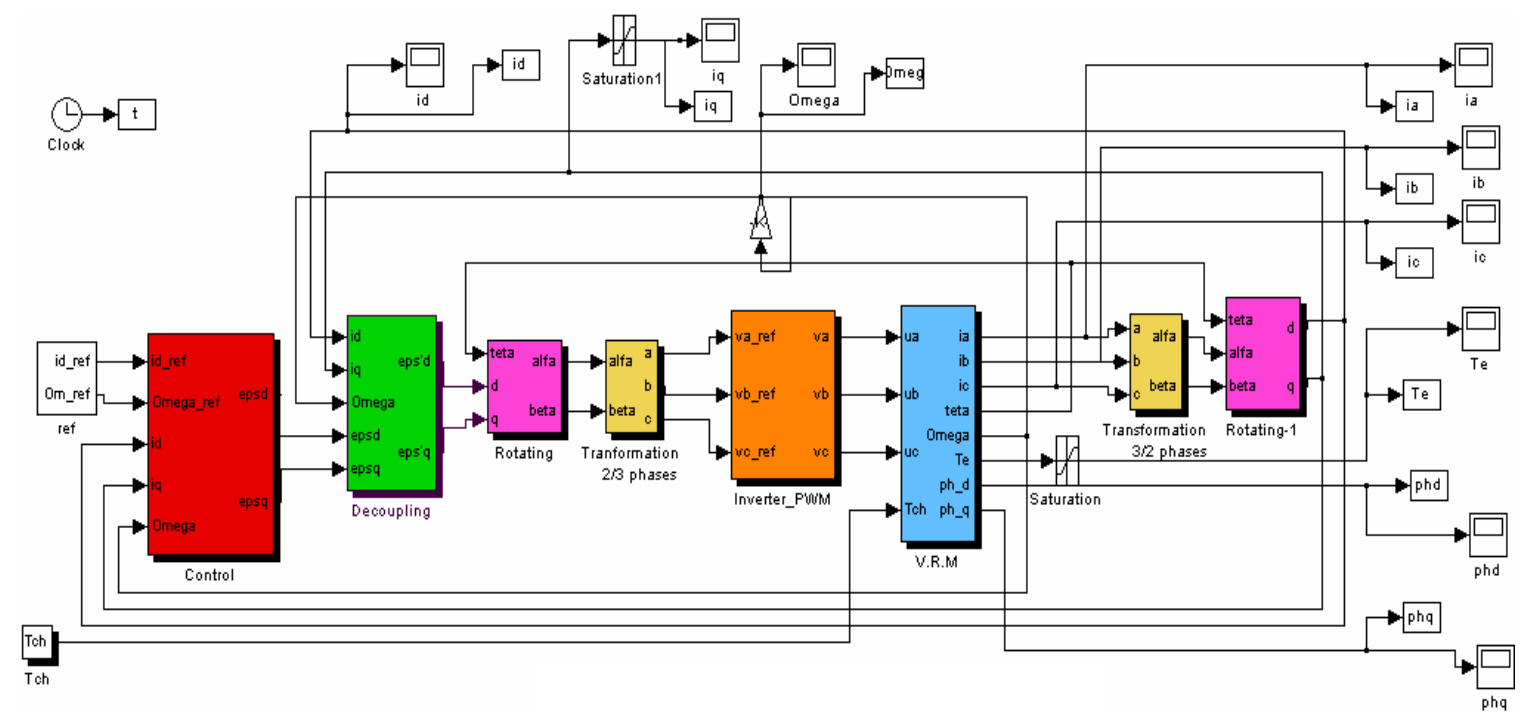

Fig. 4: Global control diagram

Vector control strategy: The principle of the vector control consists in controlling the torque while controlling instantaneously currents id and iq. The choice is to maintain the current id constant and to control the torque by iq. The variation of direct flux according to id is nonlinear where the flux in quadratic is quasi linear according to the current iq. A flux $\phi \mathrm{d}$ is then imposed only by the direct current axis id, then the torque depends only on the quadratic current $\mathrm{iq}^{[5,7]}$. But when the level of saturation is not negligible any more, taking into account the crossed effect, even if the current id is maintained constant, the values of inductances change with the load, therefore with iq. The model becomes nonlinear and useless for the control. However, we obtain a simple useful model where it can be controlled and simulated.

Structure control: The AC current machine vector control consists in measuring at every instant, the number of revolutions $\Omega$, the angular position $\theta$ and the supply currents. Once the selected value of the current id is selected, the control of the machine determines the value of the reference current iq from the loop speed $\Omega$.

The regulation of the two currents id and iq generate three-phase voltages applied to the PWM inverter. The complexity to overcome is the decoupling between both currents id and iq. This is done by the decoupling block given in the global scheme shown in Fig. 4.

\section{DISCUSSION}

The simulation of the unit (control, decoupling, converter and imbricates discs variable reluctance

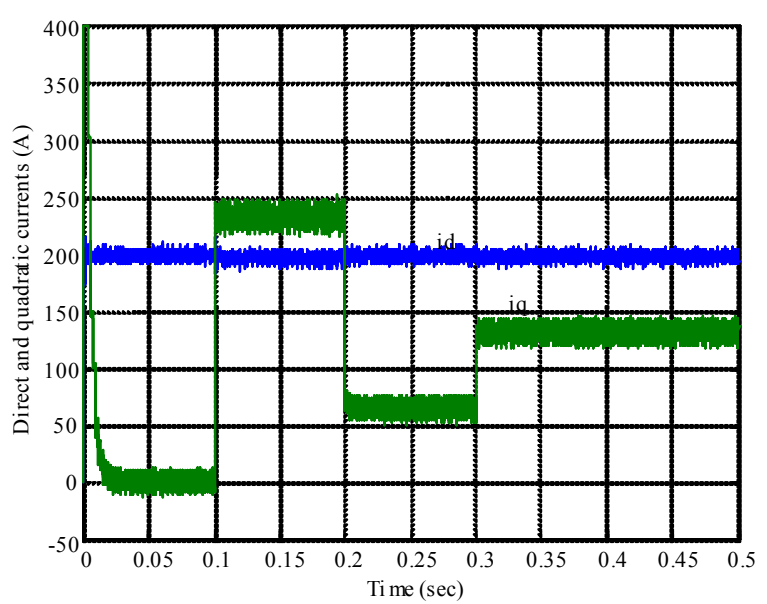

Fig. 5: Dynamical behaviour of direct and quadratic currents

machine) by (Matlab/Simulink) makes the visualisation of the various electrical and mechanical quantities possible, for some classical operations in steady state modes: starting and load torque disturbances. The machine can respond to the request with a rather small response time, then the simulation is done over a time of $0.5 \mathrm{sec}$.

When starting, the direct current id reaches quickly its reference value, this justifies the control assumption (id $=$ constant) and it maintained during the all operation .For any disturbance, the quadratic current iq reacts immediately by adopting the new adequate value, so that speed can be maintained at its reference value (Fig. 5). 


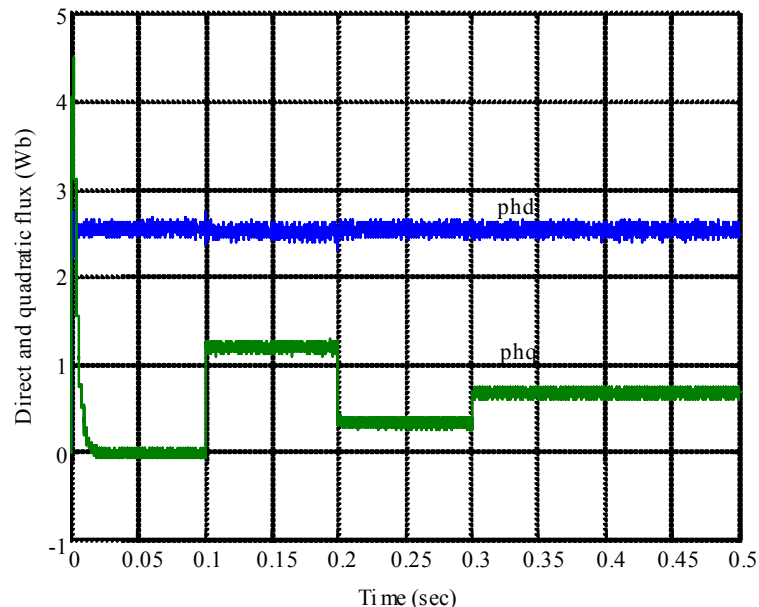

Fig. 6: Dynamical behaviour of direct and quadratic flux

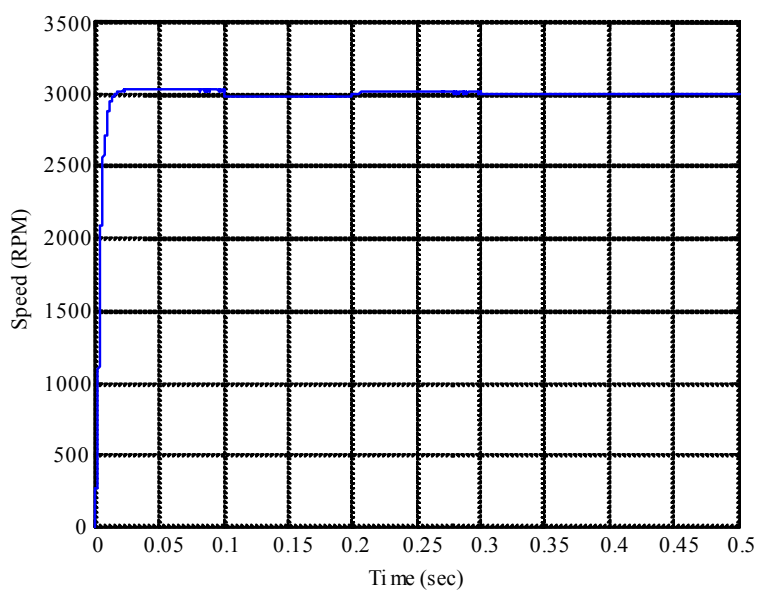

Fig. 7: Speed dynamic behaviour

The variations of direct and quadratic fluxes, which are given by Eq. 8 and 9, are shown in Fig. 6. These figures validate the proportionality between different currents and flux quantities.

Figure 7 shows the speed dynamic behaviour, where the load perturbation isn't affected.

To reach slight higher speeds:

- The increase in the inverter input voltage is possible and generate more significant voltage amplitudes. This method allows, in simulation, to reach any speed value. But, in practice that is less effective, because of the inverter over sizing and the detachment of the trapezoidal magnetic core from the Epoxy rotor under the centrifugal force ${ }^{[1]}$

- Decreasing the machine flux, i.e. decreasing the direct current id, this reduction allows reaching

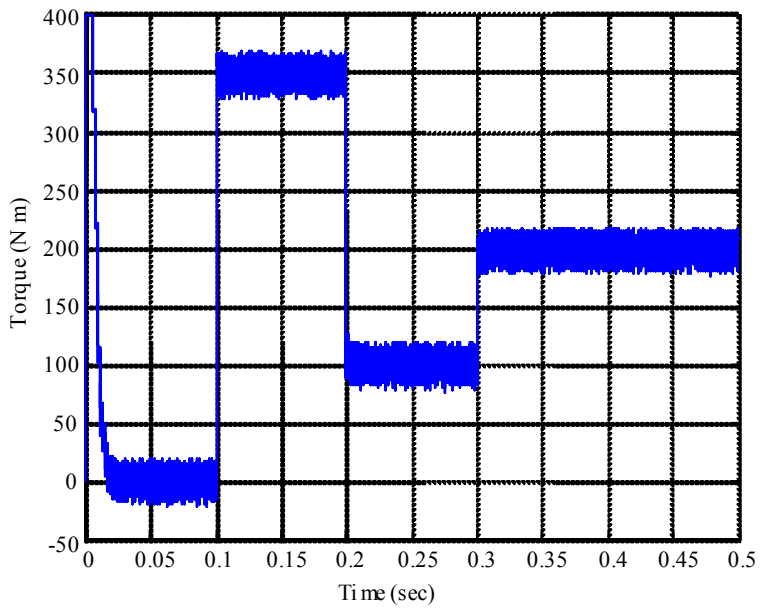

Fig. 8: Torque dynamic behaviour

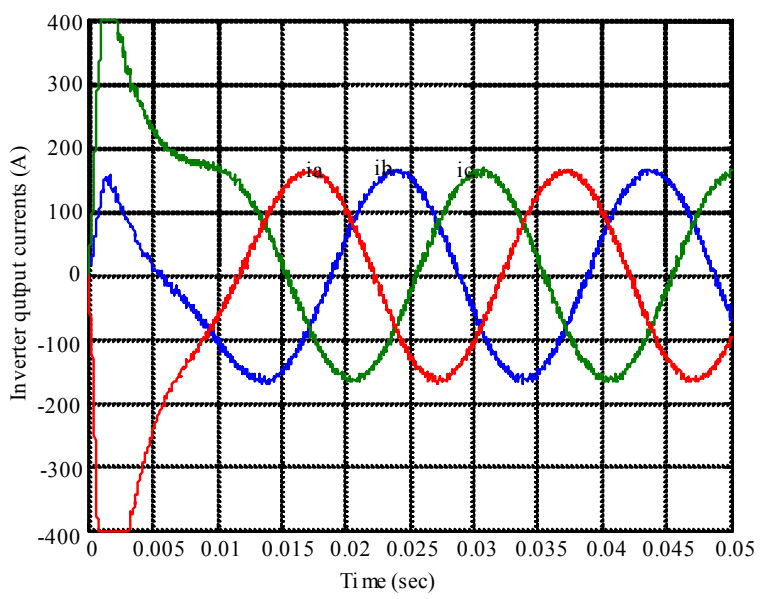

Fig. 9: Dynamic behaviour of inverter output currents

higher speeds, with the decreasing of the torque in the same proportion. The fast rise of the direct current id enables the machine to start more quickly (Fig. 7) and the fast rise of current leads to a fast rise of the torque (Fig. 8).

The adopted control strategy, using decoupling of direct id and quadratic iq currents, improves the torque dynamic, using the machine supply currents (Fig. 9).

A vector control has been simulated with the following parameters: $\mathrm{D}$-axis inductance $\mathrm{Ld}=12.3$ $[\mathrm{mH}]$, Q-axis inductance $\mathrm{Lq}=5.17[\mathrm{mH}]$, stator resistance $\mathrm{Rs}=25[\mathrm{~m} \Omega]$, inverter output voltage $\mathrm{v}=380$ [Volts], moment of inertia $\mathrm{J}=3.61\left[\mathrm{~kg} \mathrm{~m}^{2}\right]$, reference speed $\Omega$ ref $=3000$ [R.p.m], reference direct current id ref $=200[\mathrm{~A}]$. 


\section{CONCLUSION}

In this research, the imbricate discs variable reluctance machine was modelled in unsaturated mode, in which the stator construction taken identical to that of the three-phase synchronous machine. This model is very close to practical real machine, since it takes into account the effects of saturation. However it is strongly nonlinear because of the variations of inductances according to the positions of the magnetic trapezoidal core of the rotor and that of the stator.

The simulated machine is supplied by a PWM inverter, using the chopping frequency in the order of 5 $\mathrm{kHz}$, governed by the adopted control strategy (decoupling of direct and quadratic currents). This makes the dynamic improvement of the torque possible and particularly the small sensitivity of the low speed to the load disturbances.

The transformation of the single-phase voltages to the three-phase voltages and vice-versa is carried out using the blocks of transposed Concordia and Concordia transformation respectively.

This control strategy allows the control of the torque by using the quadratic current only. The method of decoupling between the direct and quadratic currents is proved very effective in the dynamic behaviour of electromagnetic torque and in rotating speed.

The results obtained by the process of decoupling are satisfactory, which makes it possible to consider a great simplification in the variable reluctance machine with stator and rotor imbricates discs control.

\section{REFERENCES}

1. E. Salame, G .Goyet, C. Lucidarne, G. Quichaud and C.Riox, 1984. Study of multidisc-reluctance motors under constant-voltage supply: Journal of Applied Physics III, 19 (7): 533-544. DOI:10.1051/rphysap: 01984001907053300
2. C. Glaize, 1985. Search forms optimal feeding of variable reluctance machines: Journal of Applied Physics III, 20 (11): 779-794. DOI: 10.1051/rphysap:019850020011077900

3. T.J.E. Miller , A. Hutton, C. Cossar and D.A. Staton, 1991. Design of a synchronous reluctance motor drive: IEEE Transaction on Industry Applications, 27(4): 741-749. DOI:10.1049/28.85491.

4. I.E. Chabu, J.R. Cardoso, V.C. Silva, S.I. Nabeta and A. Foggia, 1999. A new design technique based on a suitable choice of rotor geometrical parameters to maximize torque and power factor in synchronous reluctance motors: Part I, Theory. IEEE Transaction on Energy Conversion, 14 (3): 605-609. DOI:10.1109/60.790922.

5. A. Tounzi, T.F. Meibody and F.M. Sargos, 1997. Vector control of a smooth stator reluctance machine taking into account saturation and damping effects: Journal of Applied Physics III, France 7: 909-926. DOI: 10.1051/jp3:1997165.

6. T. Lubin, H. Razik and A. Rezzoug, 2002. Magnetic saturation effects on the control a synchronous machine: IEEE Transaction on Energy Conversion, 17 (3): 356-362. DOI: 10.1109/TEC.2002.801731.

7. B.Nahid-Mobarakeh, F. Meibody-tabar and F.M. Sargos, 2004. Mechanical sensorless control of PMSM with online estimation of stator resistance: IEEE Transaction on Industry Applications, 40 (2): 457-471. DOI:1109/TIA.2004.824490. 